\title{
Vertical and Lateral Separation
}

\author{
J. D. Proctor
}

Ir is generally assumed that the ideal distribution for the lateral or vertical distribution of aircraft on neighbouring tracks is as in Fig. I, where A and B are the centre lines of the routes or altitudes. Navaids and altimeters seem to be designed with this distribution as the ideal. Where air traffic control is imperfect and there is an appreciable risk that two aircraft without longitudinal separation will be at the same nominal altitude on the same route, then I suggest that neither the distribution of Fig. I nor that of Fig. 2 is ideal but that Fig. 3 represents the

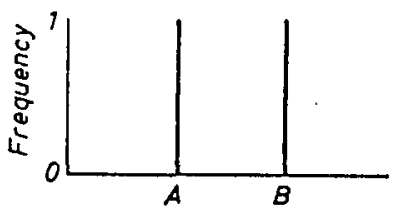

FIG. I

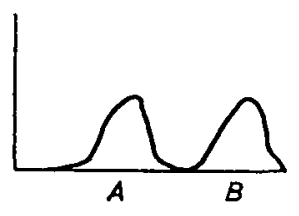

FIG. 2

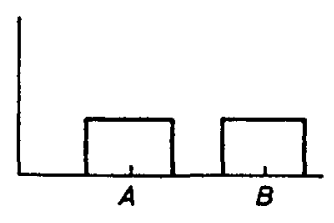

FIG. 3

ideal because it minimizes the risk of collision due to air traffic control failure. This hypothesis has implications for the design of navaids and altimeters and for the specification of vertical and lateral separation procedures.

\section{A Nocturnal}

\section{W. G. Webster}

INVESTIGATION into the design and accuracy of old-time instruments is always of interest. The Nocturnal probably used from the sixteenth century onwards for estimating the approximate local time at night is well known; with this primitive instrument the Pole Star was viewed through a central hole in a pair of wooden or brass discs. A pivoted arm turned to coincide with the 'Guards' of the Lesser Bear, the local time being deduced from an engraved scale. A seventeenthcentury improvement included an extra scale for determining the time by aligning the pointer with the Great Bear 'Pointers'.

The instrument could only have given a very approximate answer because its accuracy depended on its being held in the correct position, with the plane of the discs orthogonal to the line of sight to Polaris and, most important of all, with a small pointer on the periphery of the outer 'Calendar' scale (opposite 18 February) directed towards the observer's zenith. An error of $I^{\circ}$ in this pointer's direction would obviously have affected the answer by about 4 minutes. In addition the viewing hole seems to have been made in the centre of the discs and therefore with no allowance for the eccentricity of Polaris from the celestial pole (then about $3 \frac{1}{2}^{\circ}$ ). 